\title{
Robust potassium isotopic analysis of geological and biological samples via multicollector ICP-mass spectrometry using the 'extra-high resolution mode'
}

\author{
Kasper Hobin, Marta Costas Rodríguez, Frank Vanhaecke*
}

Ghent University, Department of Chemistry, Atomic \& Mass Spectrometry - A\&MS research unit, Campus Sterre, Krijgslaan 281 - S12, 9000 Ghent, Belgium.

* Corresponding author e-mail: frank.vanhaecke@ugent.be

\section{Supplementary information:}

- Cleaning procedure for Teflon Savillex ${ }^{\circledR}$ beakers

- Table S1, Summary of the elution protocol used for chromatographic isolation of $\mathrm{K}$ from biological and geological reference materials

- Table S2, Summary of the $\mathrm{K}$ isotope ratio results obtained in this work

- Figure S1, Long-term precision of the $\mathrm{K}$ isotope ratio as obtained from multiple measurement sessions of the in-house $\mathrm{K}$ standard solution

- Figure S2, Effect of the acidity mismatch between the sample and the bracketing standard on the $\mathrm{K}$ isotope ratio as measured in hot and cold plasma conditions

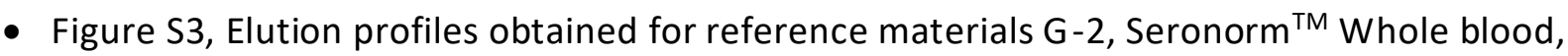
Randox laboratories LTD CSF and BCR CRM 184 Bovine muscle 


\section{Cleaning procedure for Teflon Savillex ${ }^{\circledR}$ beakers}

Teflon Savillex ${ }^{\circledR}$ beakers used for sample handling and storage were pre-cleaned with $7 \mathrm{M} \mathrm{HNO}_{3}$ and $6 \mathrm{M} \mathrm{HCl}$ at $110^{\circ} \mathrm{C}$ in several $24 \mathrm{~h}$ soaking steps and subsequently rinsed with Milli-Q water. Disposable polyethylene material was pre-cleaned by soaking in $1.0 \mathrm{M} \mathrm{HCl}$ at $110^{\circ} \mathrm{C}$ for $24 \mathrm{~h}$, followed by soaking in Milli-Q water for another $24 \mathrm{~h}$ and subsequent drying. 
Table S1. Two-stage cation exchange chromatography for $\mathrm{K}$ isolation from biological and geological samples.

\begin{tabular}{|c|c|c|c|c|c|}
\hline \multicolumn{6}{|c|}{ Geological materials } \\
\hline \multicolumn{3}{|c|}{ Step 1: AG 50W-X8 } & \multicolumn{3}{|c|}{ Step 2: AG MP-50 } \\
\hline \multicolumn{3}{|c|}{ Batch Cleaning $7 \mathrm{M} \mathrm{HCl}$} & \multicolumn{3}{|c|}{ Batch Cleaning $7 \mathrm{M} \mathrm{HCl}$} \\
\hline \multirow{3}{*}{ Cleaning } & Milli-Q (mL) & 10 & \multirow{3}{*}{ Cleaning } & Milli-Q (mL) & 10 \\
\hline & $7 \mathrm{M} \mathrm{HCl}(\mathrm{mL})$ & 10 & & $7 \mathrm{M} \mathrm{HCl}(\mathrm{mL})$ & 10 \\
\hline & Milli-Q (mL) & 10 & & Milli-Q (mL) & 10 \\
\hline Conditioning & $0.5 \mathrm{M} \mathrm{HNO}_{3}(\mathrm{~mL})$ & 10 & Conditioning & $1 \mathrm{M} \mathrm{HCl}(\mathrm{mL})$ & 10 \\
\hline Sample Load & $0.5 \mathrm{M} \mathrm{HNO}_{3}(\mathrm{~mL})$ & 5 & Sample Load & $1 \mathrm{M} \mathrm{HCl}(\mathrm{mL})$ & 1 \\
\hline Matrix & $0.5 \mathrm{M} \mathrm{HNO}_{3}(\mathrm{~mL})$ & 3 & Matrix & $1 \mathrm{M} \mathrm{HCl}(\mathrm{mL})$ & 27 \\
\hline $\mathrm{K}$ collection & $0.5 \mathrm{M} \mathrm{HNO}_{3}(\mathrm{~mL})$ & 22 & $\mathrm{~K}$ collection & $1 \mathrm{M} \mathrm{HCl}(\mathrm{mL})$ & 24 \\
\hline \multirow{2}{*}{ Cleaning } & $7 \mathrm{M} \mathrm{HCl}(\mathrm{mL})$ & 10 & \multirow{2}{*}{ Cleaning } & $7 \mathrm{M} \mathrm{HCl}(\mathrm{mL})$ & 10 \\
\hline & Milli-Q (mL) & 10 & & Milli-Q (mL) & 10 \\
\hline \multicolumn{6}{|c|}{ Biological materials } \\
\hline \multicolumn{3}{|c|}{ Step 1: AG 50W-X8 } & \multicolumn{3}{|c|}{ Step 2: AG MP-50 } \\
\hline \multicolumn{3}{|c|}{ Batch Cleaning $7 \mathrm{M} \mathrm{HCl}$} & \multicolumn{3}{|c|}{ Batch Cleaning $7 \mathrm{M} \mathrm{HCl}$} \\
\hline \multirow{3}{*}{ Cleaning } & Milli-Q (mL) & 10 & \multirow{3}{*}{ Cleaning } & Milli-Q (mL) & 10 \\
\hline & $7 \mathrm{M} \mathrm{HCl}(\mathrm{mL})$ & 10 & & $7 \mathrm{M} \mathrm{HCl}(\mathrm{mL})$ & 10 \\
\hline & Milli-Q (mL) & 10 & & Milli-Q (mL) & 10 \\
\hline Conditioning & $0.5 \mathrm{M} \mathrm{HNO}_{3}(\mathrm{~mL})$ & 10 & Conditioning & $1 \mathrm{M} \mathrm{HCl}(\mathrm{mL})$ & 10 \\
\hline Sample Load & $0.5 \mathrm{M} \mathrm{HNO}_{3}(\mathrm{~mL})$ & 5 & Sample Load & $1 \mathrm{M} \mathrm{HCl}(\mathrm{mL})$ & 1 \\
\hline Matrix & $0.5 \mathrm{M} \mathrm{HNO}_{3}(\mathrm{~mL})$ & 8 & Matrix & $1 \mathrm{M} \mathrm{HCl}(\mathrm{mL})$ & 18 \\
\hline $\mathrm{K}$ collection & $0.5 \mathrm{M} \mathrm{HNO}_{3}(\mathrm{~mL})$ & 22 & $\mathrm{~K}$ collection & $1 \mathrm{M} \mathrm{HCl}(\mathrm{mL})$ & 34 \\
\hline \multirow{2}{*}{ Cleaning } & $7 \mathrm{M} \mathrm{HCl}(\mathrm{mL})$ & 10 & \multirow{2}{*}{ Cleaning } & $7 \mathrm{M} \mathrm{HCl}(\mathrm{mL})$ & 10 \\
\hline & Milli-Q (mL) & 10 & & Milli-Q (mL) & 10 \\
\hline
\end{tabular}


Table S2. Potassium isotope ratio results for standard solutions, geological and biological reference materials. Data are expressed as $\delta^{41} \mathrm{~K}(\%)$ values relative to NIST SRM 3141a. Each reference material was analysed 3 or more times during 2-4 measurement sessions. These measurement sessions were performed on consecutive days. 2SD is two times the standard deviation of measurement replicates.

\begin{tabular}{|c|c|c|c|}
\hline & $\delta^{41} K_{\text {SRM 3141a }}$ & 2SD & $\mathbf{N}$ \\
\hline \multicolumn{4}{|l|}{ K standard solutions } \\
\hline VWR Chemicals $\mathrm{KNO}_{3}$ standard, (in-house) & -0.51 & 0.06 & 163 \\
\hline SPEX KCl standard & -0.01 & 0.04 & 7 \\
\hline \multicolumn{4}{|l|}{ Geological reference materials } \\
\hline BHVO-1 & -0.41 & 0.04 & 9 \\
\hline BHVO-2 & -0.38 & 0.04 & 8 \\
\hline BCR-1 & -0.42 & 0.06 & 11 \\
\hline DR-N & -0.55 & 0.08 & 9 \\
\hline G-2 & -0.46 & 0.08 & 7 \\
\hline \multicolumn{4}{|l|}{ Biological reference materials } \\
\hline Seronorm ${ }^{\mathrm{TM}}$ Serum & -0.34 & 0.07 & 9 \\
\hline Seronorm ${ }^{\mathrm{TM}}$ Serum & -0.29 & 0.09 & 7 \\
\hline Seronorm ${ }^{\mathrm{TM}}$ Serum & -0.28 & 0.11 & 9 \\
\hline Seronorm ${ }^{\mathrm{TM}}$ Serum & -0.30 & 0.09 & 8 \\
\hline Seronorm ${ }^{\mathrm{TM}}$ Serum & -0.30 & 0.06 & 9 \\
\hline Average & -0.30 & 0.04 & 5 \\
\hline Seronorm ${ }^{\mathrm{TM}}$ Whole blood & 0.88 & 0.10 & 7 \\
\hline Seronorm ${ }^{\mathrm{TM}}$ Whole blood & 0.88 & 0.06 & 8 \\
\hline Seronorm ${ }^{\mathrm{TM}}$ Whole blood & 0.89 & 0.08 & 9 \\
\hline Seronorm ${ }^{\mathrm{TM}}$ Whole blood & 0.90 & 0.09 & 7 \\
\hline Seronorm ${ }^{\mathrm{TM}}$ Whole blood & 0.89 & 0.07 & 8 \\
\hline Average & 0.89 & 0.02 & 5 \\
\hline Randox Cerebrospinal fluid & -0.14 & 0.02 & 9 \\
\hline Randox Cerebrospinal fluid & -0.12 & 0.04 & 9 \\
\hline Randox Cerebrospinal fluid & -0.13 & 0.03 & 7 \\
\hline Randox Cerebrospinal fluid & -0.12 & 0.03 & 9 \\
\hline Randox Cerebrospinal fluid & -0.15 & 0.03 & 8 \\
\hline Average & -0.13 & 0.03 & 5 \\
\hline TORT-3 Lobster hepatopancreas & -0.30 & 0.11 & 6 \\
\hline TORT-3 Lobster hepatopancreas & -0.31 & 0.09 & 9 \\
\hline TORT-3 Lobster hepatopancreas & -0.33 & 0.07 & 7 \\
\hline Average & -0.31 & 0.03 & 3 \\
\hline BCR-184 Bovine muscle & -0.53 & 0.03 & 9 \\
\hline BCR-184 Bovine muscle & -0.51 & 0.03 & 8 \\
\hline BCR-184 Bovine muscle & -0.53 & 0.04 & 8 \\
\hline BCR-184 Bovine muscle & -0.52 & 0.03 & 9 \\
\hline BCR-184 Bovine muscle & -0.51 & 0.06 & 9 \\
\hline Average & -0.52 & 0.02 & 5 \\
\hline
\end{tabular}




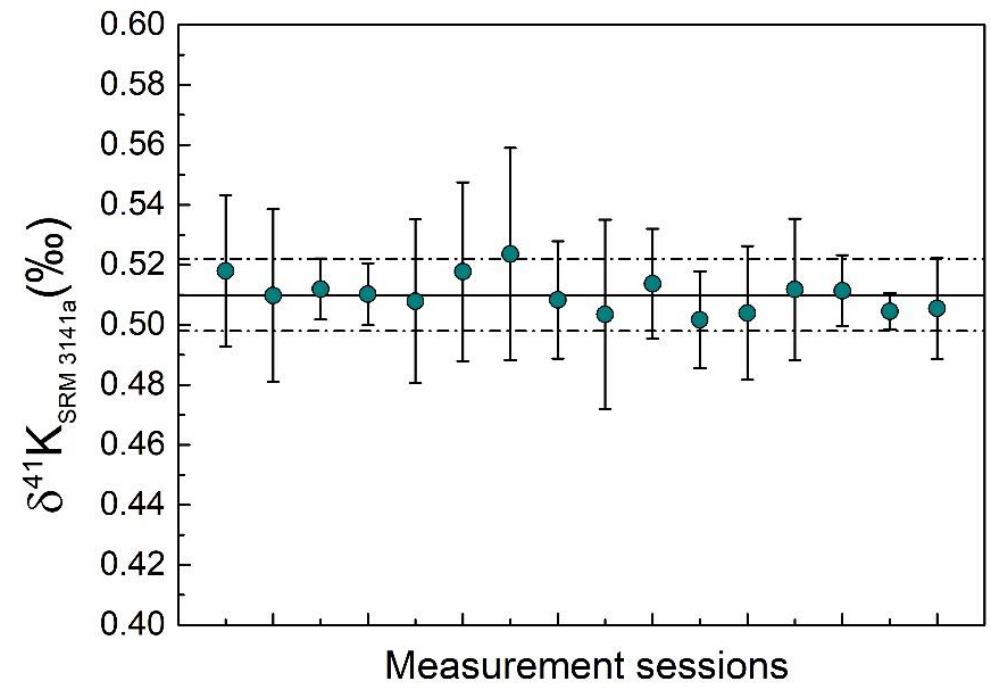

Figure S1. Long-term precision of the $\mathrm{K}$ isotope ratio obtained from multiple measurement sessions of the in-house $\mathrm{K}$ standard. Each data point represents the average $\delta^{41} \mathrm{~K}_{\mathrm{SRM}} 3141 \mathrm{a}$ value of one measurement session and error bars indicate the 2SD of the measurement replicates $(\mathrm{N}=5-33)$ within each measurement session. The $\delta^{41} \mathrm{~K}_{\mathrm{SRM} 3141 \mathrm{a}}$ value of the in-house $\mathrm{K}$ standard obtained over 8 months was $0.51 \pm 0.01 \%$ o (2SD, $N=16)$. 


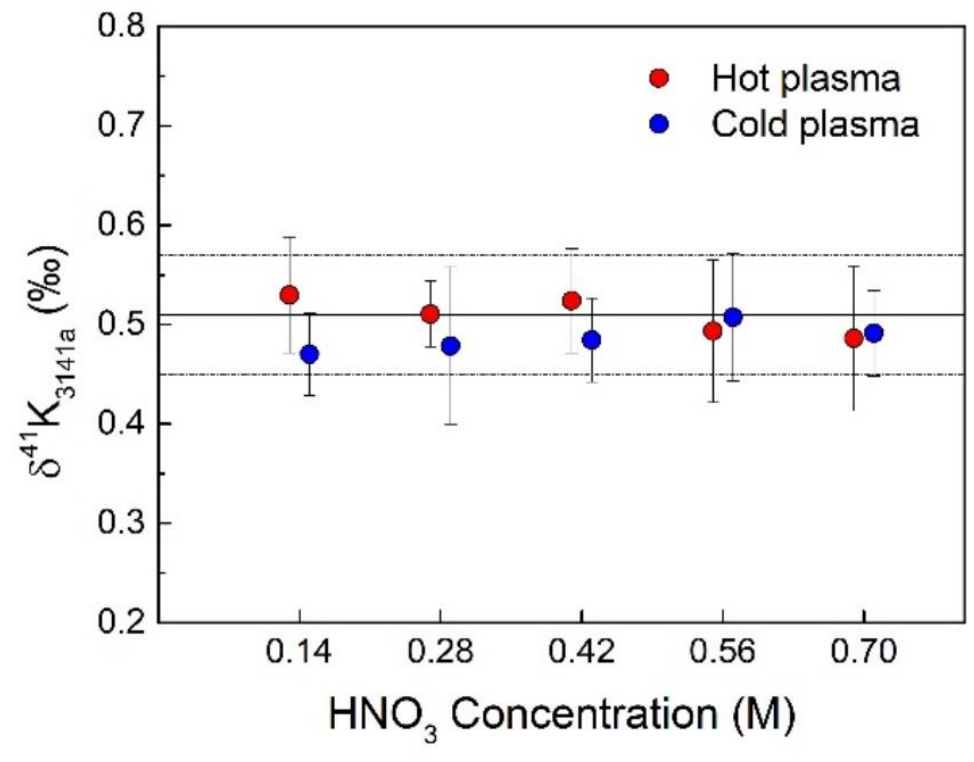

Figure S2. Effect of the acidity mismatch between the sample and the bracketing standard on the $\mathrm{K}$ isotope ratio as measured in hot and cold plasma conditions. 

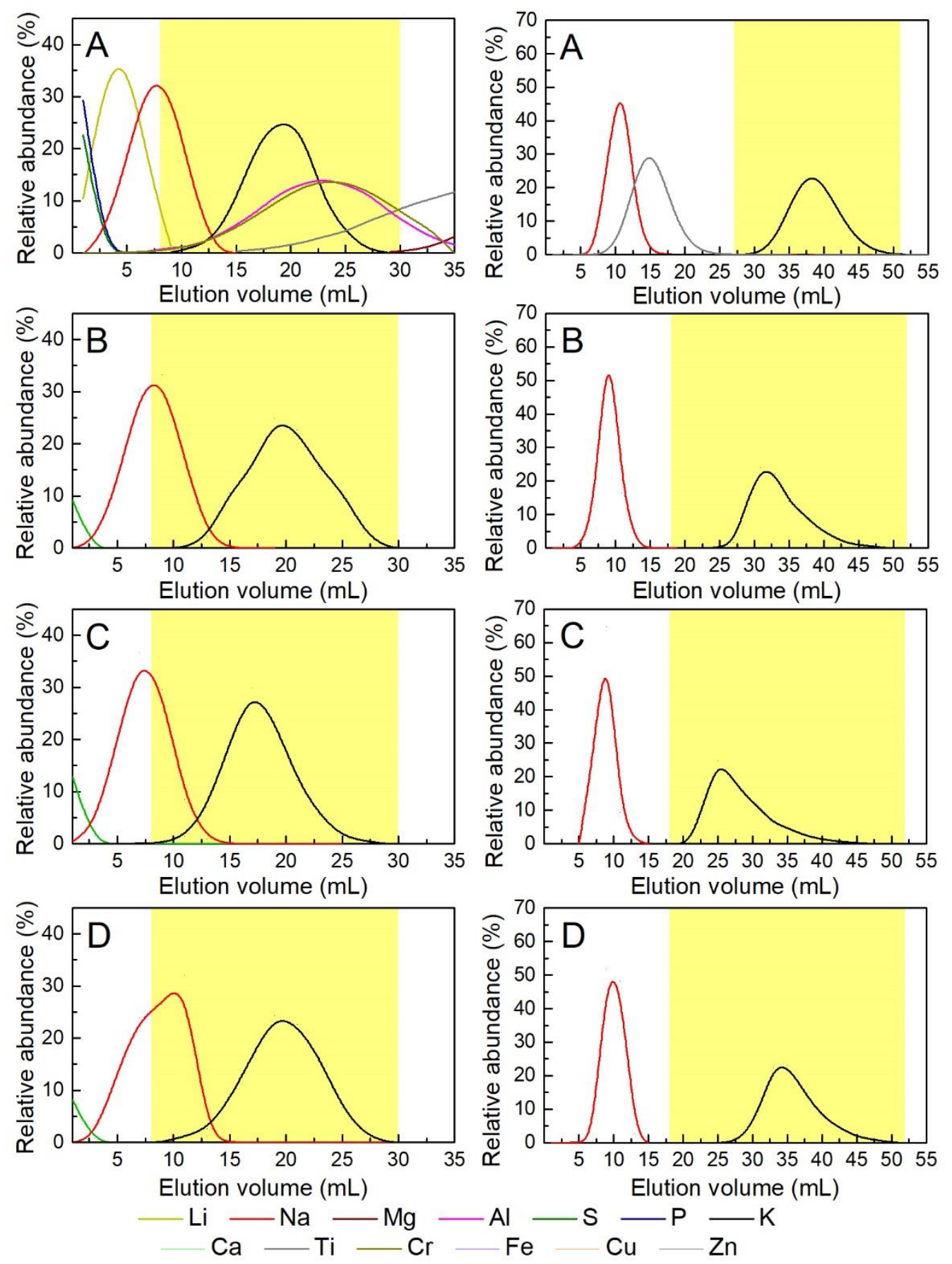

Figure S3. Elution profiles for the reference materials G-2 (A), Seronorm ${ }^{\mathrm{TM}}$ Whole blood (B), Randox laboratories LTD CSF (C) and BCR CRM 184 Bovine muscle (D). Matrix elements monitored were $\mathrm{Li}, \mathrm{Na}, \mathrm{Mg}, \mathrm{Al}, \mathrm{S}, \mathrm{P}, \mathrm{K}, \mathrm{Ca}, \mathrm{Ti}, \mathrm{Cr}, \mathrm{Fe}, \mathrm{Cu}$ and $\mathrm{Zn}$. 